\title{
Linear complexity and autocorrelation of a new class of binary generalized cyclotomic sequences of order two and length $p q r$
}

\author{
JIA Wenjuan ${ }^{1}$, GAO Juntao ${ }^{2, *}$, and ZHANG Peng ${ }^{1}$ \\ 1. School of Telecommunications Engineering, Xidian University, Xi' an 710071, China; \\ 2. The State Key Laboratory of Integrated Services Network, Xidian University, Xi' an 710071, China
}

\begin{abstract}
Cyclotomic sequences have good cryptographic properties and are closely related to difference sets. This paper proposes a new class of binary generalized cyclotomic sequences of order two and length $p q r$. Its linear complexity, minimal polynomial, and autocorrelation are investigated. The results show that these sequences have a large linear complexity when $2 \in D_{1}$, which means they can resist the Berlekamp-Massey attack. Furthermore, the autocorrelation values are close to 0 with a probability of approximately $1-1 / r$. Therefore, when $r$ is a big prime, the new sequence has a good autocorrelation.
\end{abstract}

Keywords: generalized cyclotomic sequence, linear complexity, minimal polynomial, autocorrelation value.

DOI: $10.21629 /$ JSEE.2019.04.03

\section{Introduction}

The linear complexity of Legendre sequences was determined in [1,2]. In 1962, Whiteman introduced the generalized cyclotomy [3], where the motivation was to search for difference sets. Based on the Whiteman's generalized cyclotomy, Ding constructed generalized cyclotomic sequences of order two [4], and gave its linear complexity and autocorrelation in $[5,6]$. More works about linear complexities and autocorrelation values of Whiteman's generalized cyclotomic sequences can be referred to [7,8]. Later, a new generalized cyclotomy with respect to $p_{1}^{e_{1}} p_{2}^{e_{2}} \cdots p_{t}^{e_{t}}$ was introduced by Ding and Helleseth [9].

Shortly after, based on Ding and Helleseth's generalized cyclotomic class, the new generalized cyclotomic sequence of order two and order four were constructed and their pseudorandom properties were studied in [10-12]. Many researchers have focused on the linear complexity

\footnotetext{
Manuscript received July 30, 2018.

*Corresponding author

This work was supported by the National Key Research and Development Program of China (2016YFB0800601), the Natural Science Foundation of China (61303217; 61502372), the Fundamental Research Funds for the Central Universities (JB140115), and the Natural Science Foundation of Shaanxi Province (2013JQ8002; 2014JQ8313).
}

and the autocorrelation values of generalized cyclotomic sequences with different characteristic sets, orders, and periods [13-23]. Moreover, some polyphase generalized cyclotomic sequences were studied in [24-26].

Chang et al. and Liu et al. constructed a new class of generalized cyclotomic sequences with respect to $p q r$ over $G F(2)$, and determined its linear complexity with the cyclotomic theory. They showed that these sequences have a high linear complexity $[27,28]$. LYU et al. studied the linear complexity of a new generalized cyclotomic sequence of order two over $\mathbb{Z}_{p_{1} p_{2} p_{3}}$ [29].

This paper proposes a new class of binary generalized cyclotomic sequences of order two and the length $N=p q r$, and determines their linear complexities, minimal polynomials, and autocorrelation values. The new sequences have a different characteristic set from that in [2729]. We show that the sequences have high linear complexity when $2 \in D_{1}$. Besides, we determine the minimal polynomial of the new sequences by calculating the great common divisor of $x^{N}-1$ and $S(x)$. Moreover, the autocorrelation values are determined by considering properties of the quadratic character in finite fields.

Linear complexity and autocorrelation are very important indicators for measuring the security of sequences used in cryptography. The linear complexity $L C\left(z_{i}\right)$ of a $T$-periodic binary sequence $\left(z_{i}\right)$ is defined as the length of the shortest linear feedback shift register (LFSR) which can generate the sequence. More specifically, the linear complexity is the smallest positive integer $L$ satisfying

$$
z_{i}=c_{1} z_{i-1}+c_{2} z_{i-2}+\cdots+c_{L} z_{i-L}
$$

where $c_{1}, c_{2}, \ldots, c_{L} \in G F(2)$ and $i \geqslant L$. The generating polynomial of $\left(z_{i}\right)$ is defined by

$$
Z(x)=z_{0}+z_{1} x+\cdots+z_{T-1} x^{T-1} .
$$

It is well-known that the minimal polynomial of $\left(z_{i}\right)$ is $m(x)=\left(x^{T}-1\right) / \operatorname{gcd}\left(x^{T}-1, Z(x)\right)$, and the linear com- 
plexity of $\left(z_{i}\right)$ is $L C\left(z_{i}\right)=T-\operatorname{deg}\left(\operatorname{gcd}\left(x^{T}-1, Z(x)\right)\right)$ (see [22, Lemma 8.2.1]). A low linear complexity (compared with $T / 2$, where $T$ denotes the period of the sequence) is not desirable for unpredictability of sequences in cryptography: if the linear complexity is $l$, then $2 l$ consecutive bits of the sequence could be used to determine the whole sequence by using the Berlekamp-Massey algorithm.

The autocorrelation function of a binary sequence $\left(z_{i}\right)$ with the period $T$ is defined as

$$
A C\left(z_{i}, \tau\right)=\frac{1}{T} \sum_{i \in \mathrm{Z}_{T}}(-1)^{z_{i}+z_{i+\tau}}
$$

where $0 \leqslant \tau \leqslant T-1$. Only when the values of $A C\left(z_{i}, \tau\right)$ distribute flat and low, the sequence $\left(z_{i}\right)$ is easy to distinguish from each time shifted version of itself [18]. The autocorrelation function with the ideal distribution of values is two-valued, which is given by

$$
A C\left(z_{i}, \tau\right)=\left\{\begin{array}{l}
1, \tau=0 \\
1 / T, \quad \text { otherwise }
\end{array} .\right.
$$

This paper is scheduled as follows. First we give the definition of new binary generalized cyclotomic sequences $\left(s_{i}\right)$ in Section 2. In Section 3, we calculate the linear complexity and the minimal polynomial of $\left(s_{i}\right)$. The results show that the sequence has a high linear complexity when $2 \in D_{1}$. In Section 4, we study the autocorrelation values of $\left(s_{i}\right)$. In Section 5, we give the simulation and conclusion is made in Section 6.

\section{Generalized cyclotomic sequences}

Let $p, q$ and $r$ be odd primes with $d=\operatorname{gcd}(p-1, q-1)=$ $\operatorname{gcd}(p-1, r-1)=\operatorname{gcd}(q-1, r-1)=2$. By the Chinese remainder theorem, there exists a common primitive root $g$ of $p, q$ and $r$. Put $N=p q r, e=(p-1)(q-1)(r-1) / 4$, and two positive integers $x_{1}$ and $x_{2}$ satisfy

$$
\left\{\begin{array} { l } 
{ x _ { 1 } \equiv g ( \operatorname { m o d } p ) } \\
{ x _ { 1 } \equiv 1 ( \operatorname { m o d } q ) } \\
{ x _ { 1 } \equiv 1 ( \operatorname { m o d } r ) }
\end{array} \left\{\begin{array}{l}
x_{2} \equiv 1(\bmod p) \\
x_{2} \equiv g(\bmod q) \\
x_{2} \equiv 1(\bmod r)
\end{array} .\right.\right.
$$

Define

$$
\begin{gathered}
D_{0}=\left\{g^{2 s}, g^{2 s} x_{1}, g^{2 s} x_{2}, g^{2 s} x_{1} x_{2}: s=0,1, \ldots, \frac{e}{2}-1\right\} \\
D_{1}=\left\{g^{2 s+1}, g^{2 s+1} x_{1}, g^{2 s+1} x_{2},\right. \\
\left.g^{2 s+1} x_{1} x_{2}: s=0,1, \ldots, \frac{e}{2}-1\right\} .
\end{gathered}
$$

We have $\mathbb{Z}_{N}^{*}=D_{0} \cup D_{1}$ and $D_{0} \cap D_{1}=\varnothing$, where $\varnothing$ denotes the empty set. Define

$$
P=\{p, 2 p, \ldots,(q r-1) p\}
$$

$$
\begin{gathered}
P_{1}=\{p q, 2 p q, \ldots,(r-1) p q\} \\
P_{2}=\{p r, 2 p r, \ldots,(q-1) p r\} \\
P_{3}=P-P_{1}-P_{2} .
\end{gathered}
$$

Furthermore, we divide $P_{1}, P_{2}$ and $P_{3}$ into two subsets respectively, that is

$$
\begin{gathered}
D_{0}^{(r)}=\left\{g^{2 s} \bmod r: s=0,1, \ldots, \frac{r-1}{2}-1\right\} \\
D_{1}^{(r)}=\left\{g^{2 s+1} \bmod r: s=0,1, \ldots, \frac{r-1}{2}-1\right\} \\
P_{10}=p q D_{0}^{(r)}, \quad P_{11}=p q D_{1}^{(r)}, \quad P_{1}=P_{10} \cup P_{11} \\
D_{0}^{(q)}=\left\{g^{2 s} \bmod q: s=0,1, \ldots, \frac{q-1}{2}-1\right\} \\
D_{1}^{(q)}=\left\{g^{2 s+1} \bmod q: s=0,1, \ldots, \frac{q-1}{2}-1\right\} \\
P_{20}=p r D_{0}^{(q)}, \quad P_{21}=p r D_{1}^{(q)}, P_{2}=P_{20} \cup P_{21} \\
D_{0}^{(q r)}=\left\{g^{2 s} \bmod q r, g^{2 s} x \bmod q r: s=\right. \\
\left.0,1, \ldots, \frac{e_{q r}}{2}-1\right\} \\
D_{1}^{(q r)}=\left\{g^{2 s+1} \bmod q r, g^{2 s+1} x \bmod q r: s=\right. \\
P_{30}=p D_{0}^{(q r)}, \quad P_{31}=p D_{1}^{(q r)}, \quad P_{3}=P_{30} \cup P_{31}
\end{gathered}
$$

where $e_{q r}=\frac{(q-1)(r-1)}{2}, x \equiv g(\bmod q)$ and $x \equiv$ $1(\bmod r)$, then

$$
\begin{gathered}
\mathbb{Z}_{r}^{*}=D_{0}^{(r)} \cup D_{1}^{(r)}, D_{0}^{(r)} \cap D_{1}^{(r)}=\varnothing \\
\mathbb{Z}_{q}^{*}=D_{0}^{(q)} \cup D_{1}^{(q)}, D_{0}^{(q)} \cap D_{1}^{(q)}=\varnothing \\
\mathbb{Z}_{q r}^{*}=D_{0}^{(q r)} \cup D_{1}^{(q r)}, D_{0}^{(q r)} \cap D_{1}^{(q r)}=\varnothing .
\end{gathered}
$$

Define

$$
\begin{gathered}
Q=\{q, 2 q, \ldots,(p r-1) q\} \\
Q_{1}=\{p q, 2 p q, \ldots,(r-1) p q\}=P_{1} \\
Q_{2}=\{q r, 2 q r, \ldots,(p-1) q r\} \\
Q_{3}=Q-Q_{1}-Q_{2} \\
R=\{r, 2 r, \ldots,(p q-1) r\}-P_{2}-Q_{2} .
\end{gathered}
$$

Note that we divide $Q_{2}, Q_{3}$ and $R$ into two subsets respectively, that is,

$$
\begin{gathered}
D_{0}^{(p)}=\left\{g^{2 s} \bmod p: s=0,1, \ldots, \frac{p-1}{2}-1\right\} \\
D_{1}^{(p)}=\left\{g^{2 s+1} \bmod p: s=0,1, \ldots, \frac{p-1}{2}-1\right\} \\
Q_{20}=q r D_{0}^{(p)}, \quad Q_{21}=q r D_{1}^{(p)}, \quad Q_{2}=Q_{20} \cup Q_{21}
\end{gathered}
$$




$$
\begin{gathered}
D_{0}^{(p r)}=\left\{g^{2 s} \bmod p r, g^{2 s} y \bmod p r: s=\right. \\
\left.0,1, \ldots, \frac{e_{p r}}{2}-1\right\} \\
D_{1}^{(p r)}=\left\{g^{2 s+1} \bmod p r, g^{2 s+1} y \bmod p r: s=\right. \\
\left.0,1, \ldots, \frac{e_{p r}}{2}-1\right\} \\
Q_{30}=q D_{0}^{(p r)}, \quad Q_{31}=q D_{1}^{(p r)}, \quad Q_{3}=Q_{30} \cup Q_{31} \\
D_{0}^{(p q)}=\left\{g^{2 s} \bmod p q, g^{2 s} z \bmod p q: s=\right. \\
\left.0,1, \ldots, \frac{e_{p q}}{2}-1\right\} \\
D_{1}^{(p q)}=\left\{g^{2 s+1} \bmod p q, g^{2 s+1} z \bmod p q: s=\right. \\
\left.0,1, \ldots, \frac{e_{p q}}{2}-1\right\} \\
R_{0}=r D_{0}^{(p q)}, \quad R_{1}=r D_{1}^{(p q)}, \quad R=R_{0} \cup R_{1} \\
O=\{0\}
\end{gathered}
$$

where $e_{p r}=\frac{(p-1)(r-1)}{2}, y \equiv g(\bmod p)$ and $y \equiv$

$1(\bmod r), e_{p q}=\frac{(p-1)(q-1)}{2}, z \equiv g(\bmod p)$ and $z \equiv$ $1(\bmod q)$, then

$$
\begin{array}{cl}
\mathbb{Z}_{p}^{*}=D_{0}^{(p)} \cup D_{1}^{(p)}, & D_{0}^{(p)} \cap D_{1}^{(p)}=\varnothing \\
\mathbb{Z}_{p r}^{*}=D_{0}^{(p r)} \cup D_{1}^{(p r)}, & D_{0}^{(p r)} \cap D_{1}^{(p r)}=\varnothing \\
\mathbb{Z}_{p q}^{*}=D_{0}^{(p q)} \cup D_{1}^{(p q)}, & D_{0}^{(p q)} \cap D_{1}^{(p q)}=\varnothing .
\end{array}
$$

We have $\mathbb{Z}_{N}=D_{0} \cup D_{1} \cup P \cup Q_{2} \cup Q_{3} \cup R \cup O$. Define $C_{0}=D_{0} \cup Q_{3} \cup R \cup O, C_{1}=D_{1} \cup P \cup Q_{2}$.

Based on the generalized cyclotomy, the new binary generalized cyclotomic sequence $\left(s_{i}\right)$ of order two and the length $p q r$ over $G F(2)$ is defined by

$$
s_{i}= \begin{cases}0, & i \bmod N \in C_{0} \\ 1, & i \bmod N \in C_{1}\end{cases}
$$

where $i \geqslant 0$.

\section{Linear complexity and minimal polynomial}

The generating polynomial of $\left(s_{i}\right)$ is defined as $S(x)=$ $\sum_{i \in C_{1}} x^{i}=\sum_{i \in D_{1}} x^{i}+\sum_{i \in P} x^{i}+\sum_{i \in Q_{2}} x^{i} \in G F(2)[x]$. Let $\alpha$ be a primitive $N$ th root of unit over the field $G F\left(2^{m}\right)$, where $m=\operatorname{ord}_{N}(2)$, we have

$$
L C\left(s_{i}\right)=N-\left|\left\{j: S\left(\alpha^{j}\right)=0,0 \leqslant j \leqslant N-1\right\}\right| .
$$

If $x=\alpha^{0}=1$, we have

$$
S(1)=\sum_{i \in D_{1}} 1+\sum_{i \in P} 1+\sum_{i \in Q_{2}} 1 \equiv 0(\bmod 2) .
$$

Lemma 1 [28] Over the ring of $G F(2)[x], \sum_{i \in \mathbb{Z}_{N}^{*}} \alpha^{i}=$ $\sum_{i \in P} \alpha^{i}=\sum_{i \in Q} \alpha^{i}=\sum_{i \in R} \alpha^{i}=1, \sum_{i \in P_{1}} \alpha^{i}=\sum_{i \in P_{2}} \alpha^{i}=$ $\sum_{i \in P_{3}} \alpha^{i}=1, \sum_{i \in Q_{2}} \alpha^{i}=\sum_{i \in Q_{3}} \alpha^{i}=1$.

Lemma 2 [30] If $a \in D_{j}$, then $a D_{i}=D_{(i+j)(\bmod 2)}$ $(i, j=0,1)$.

Lemma 3 Let the symbols be the same as before, then

$$
S\left(\alpha^{k}\right)=\left\{\begin{array}{l}
S(\alpha), \quad k \in D_{0} \\
1+S(\alpha), \quad k \in D_{1} \\
1, \quad k \in P \cup Q_{2} \\
0, \quad k \in O \cup Q_{3} \\
\frac{r-1}{2}(\bmod 2), \quad k \in R
\end{array} .\right.
$$

Proof If $k \in D_{0}$, by Lemma $2, k P=P, k Q_{2}=Q_{2}$ and $k D_{1}=D_{1}$, then

$$
\begin{gathered}
S\left(\alpha^{k}\right)=\sum_{i \in D_{1}} \alpha^{k i}+\sum_{i \in P} \alpha^{k i}+\sum_{i \in Q_{2}} \alpha^{k i}= \\
\sum_{i \in D_{1}} \alpha^{i}+\sum_{i \in P} \alpha^{i}+\sum_{i \in Q_{2}} \alpha^{i}=S(\alpha) .
\end{gathered}
$$

If $k \in D_{1}$, by Lemma $2, k P=P, k Q_{2}=Q_{2}$ and $k D_{1}=D_{0}$, then

$$
\begin{gathered}
S\left(\alpha^{k}\right)=\sum_{i \in D_{1}} \alpha^{k i}+\sum_{i \in P} \alpha^{k i}+\sum_{i \in Q_{2}} \alpha^{k i}= \\
\sum_{i \in D_{0}} \alpha^{i}+\sum_{i \in P} \alpha^{i}+\sum_{i \in Q_{2}} \alpha^{i}=1+S(\alpha) .
\end{gathered}
$$

(i) If $k \in P_{1}$, we get

$D_{1} \bmod r=\left\{g^{2 s+1} \bmod r: s=0,1, \ldots, \frac{e}{2}-1\right\}=D_{1}^{(r)}$, when $s$ ranges over $\left\{0,1, \ldots, \frac{e}{2}-1\right\}$, and $D_{1} \bmod r$ runs through $D_{1}^{(r)}(p-1)(q-1)$ times, then

$$
\sum_{i \in D_{1}} \alpha^{k i}=((p-1)(q-1) \bmod 2) \sum_{i \in P_{11}} \alpha^{i}=0 .
$$

Since $k P=P_{1} \cup O$, we have

$\sum_{i \in P} \alpha^{k i}=(q \bmod 2) \sum_{i \in P_{1}} \alpha^{i}+(q-1) \bmod 2=1$.

Since $k Q_{2}=O$, we have

$$
\sum_{i \in Q_{2}} \alpha^{k i}=(p-1) \bmod 2=0 .
$$

Then

$$
S\left(\alpha^{k}\right)=\sum_{i \in D_{1}} \alpha^{k i}+\sum_{i \in P} \alpha^{k i}+\sum_{i \in Q_{2}} \alpha^{k i}=1 .
$$


(ii) If $k \in P_{2}$, we get

$D_{1} \bmod q=\left\{g^{2 s} \bmod q, g^{2 s+1} \bmod q: s=\right.$

$$
\left.0,1, \ldots, \frac{e}{2}-1\right\}=\mathbb{Z}_{q}^{*},
$$

when $s$ ranges over $\left\{0,1, \ldots, \frac{e}{2}-1\right\}$, and $D_{1} \bmod q$ runs through $\mathbb{Z}_{q}^{*} \frac{(p-1)(r-1)}{2}$ times, then

$$
\sum_{i \in D_{1}} \alpha^{k i}=\left(\frac{(p-1)(r-1)}{2} \bmod 2\right) \sum_{i \in P_{2}} \alpha^{i}=0 .
$$

Since $k P=P_{2} \cup O$, we have

$$
\sum_{i \in P} \alpha^{k i}=(r \bmod 2) \sum_{i \in P_{2}} \alpha^{i}+(r-1) \bmod 2=1 \text {. }
$$

Since $k Q_{2}=O$, we have

$$
\sum_{i \in Q_{2}} \alpha^{k i}=(p-1) \bmod 2=0 .
$$

Then

$$
S\left(\alpha^{k}\right)=\sum_{i \in D_{1}} \alpha^{k i}+\sum_{i \in P} \alpha^{k i}+\sum_{i \in Q_{2}} \alpha^{k i}=1 .
$$

(iii) If $k \in P_{3}$, we get

$D_{1} \bmod q r=\left\{g^{2 s+1} \bmod q r, g^{2 s+1} x_{2} \bmod q r: s=\right.$

$$
\left.0,1, \ldots, \frac{e}{2}-1\right\}=D_{1}^{(q r)}
$$

when $s$ ranges over $\left\{0,1, \ldots, \frac{e}{2}-1\right\}$, and $D_{1} \bmod q r$ runs through $D_{1}^{(q r)} p-1$ times, then

$$
\sum_{i \in D_{1}} \alpha^{k i}=((p-1) \bmod 2) \sum_{i \in P_{31}} \alpha^{i}=0 .
$$

Since $k P=P$, we have

$$
\sum_{i \in P} \alpha^{k i}=\sum_{i \in P} \alpha^{i}=1
$$

Since $k Q_{2}=O$, we have

$$
\sum_{i \in Q_{2}} \alpha^{k i}=(p-1) \bmod 2=0 .
$$

Then

$$
S\left(\alpha^{k}\right)=\sum_{i \in D_{1}} \alpha^{k i}+\sum_{i \in P} \alpha^{k i}+\sum_{i \in Q_{2}} \alpha^{k i}=1 .
$$

From (i), (ii) and (iii), we have $S\left(\alpha^{k}\right)=1$ when $k \in P$. Similarly, we have $S\left(\alpha^{k}\right)=1$ when $k \in Q_{2}$, and $S\left(\alpha^{k}\right)=1$ when $k \in Q_{3}$. (iv) If $k \in O$, we have

$$
S\left(\alpha^{k}\right)=S(1)=0 .
$$

(v) If $k \in R$, we have

$$
D_{1} \bmod p q=\mathbb{Z}_{p q}^{*},
$$

when $s$ ranges over $\left\{0,1, \ldots, \frac{e}{2}-1\right\}$, and $D_{1} \bmod p q$ runs through $\mathbb{Z}_{p q}^{*} \frac{r-1}{2}$ times, then

$$
\sum_{i \in D_{1}} \alpha^{k i}=\left(\frac{r-1}{2} \bmod 2\right) \sum_{i \in R} \alpha^{i}=\frac{r-1}{2}(\bmod 2) .
$$

Since $k P=P_{2} \cup O$, we have

$\sum_{i \in P} \alpha^{k i}=(r \bmod 2) \sum_{i \in P_{2}} \alpha^{i}+(r-1) \bmod 2=1$.

Since $k Q_{2}=Q_{2}$, we have

$$
\sum_{i \in Q_{2}} \alpha^{k i}=\sum_{i \in Q_{2}} \alpha^{i}=1 \text {. }
$$

Then

$$
S\left(\alpha^{k}\right)=\sum_{i \in D_{1}} \alpha^{k i}+\sum_{i \in P} \alpha^{k i}+\sum_{i \in Q_{2}} \alpha^{k i}=\frac{r-1}{2}(\bmod 2) .
$$

Lemma $4 S(\alpha) \in\{0,1\}$ if and only if $2 \in D_{0}$.

Proof Since the characteristic of the finite field $G F\left(2^{m}\right)$ is 2 , it follows that $S^{2}(\alpha)=S\left(\alpha^{2}\right)$. By Lemma 3 , we have $S\left(\alpha^{2}\right)=S(\alpha)$ if and only if $2 \in D_{0}$.

Theorem 1 Let notations be the same as before, then the linear complexity $L C\left(s_{i}\right)$ of the binary generalized cyclotomic sequence $\left(s_{i}\right)$ satisfies

(i) If $r \equiv 1 \bmod 4$,

$L C\left(s_{i}\right)=\left\{\begin{array}{l}p q r-\frac{(p-1)(q-1)(r-1)}{2}-\delta, \quad 2 \in D_{0} \\ p q r-\delta, \quad 2 \in D_{1}^{2}\end{array}\right.$

where $\delta=(p-1)(q-1)+(p-1)(r-1)+1$.

(ii) If $r \equiv 3 \bmod 4$,

$L C\left(s_{i}\right)=\left\{\begin{array}{l}p q r-\frac{(p-1)(q-1)(r-1)}{2}-\eta, \quad 2 \in D_{0} \\ p q r-\eta, \quad 2 \in D_{1}^{2}\end{array}\right.$ where $\eta=(p-1)(r-1)+1$.

Proof (i) If $r \equiv 1 \bmod 4$, and $2 \in D_{0}$, by Lemma 3, only one of $S(\alpha)$ and $1+S(\alpha)$ equals 0 , here we choose $\alpha$ such that $S(\alpha)=0$. Thus,

$$
S\left(\alpha^{k}\right)=\left\{\begin{array}{ll}
0, & k \in D_{0} \\
1, & k \in D_{1} \\
1, & k \in P \cup Q_{2} \\
0, & k \in O \cup Q_{3} \cup R
\end{array} .\right.
$$


Combined with (2), we get

$$
\begin{gathered}
L C\left(s_{i}\right)=p q r-\frac{(p-1)(q-1)(r-1)}{2}-1- \\
(p-1)(r-1)-(p-1)(q-1)= \\
p q r-\frac{(p-1)(q-1)(r-1)}{2}-\delta .
\end{gathered}
$$

If $r \equiv 1 \bmod 4$, and $2 \in D_{1}$, by Lemma 3 and Lemma 4, we have

$$
S\left(\alpha^{k}\right)\left\{\begin{array}{ll}
\neq 0, & k \in D_{0} \\
\neq 0, & k \in D_{1} \\
=1, & k \in P \cup Q_{2} \\
=0, & k \in O \cup Q_{3} \cup R
\end{array} .\right.
$$

Hence, $L C\left(s_{i}\right)=p q r-\delta$.

(ii) If $r \equiv 3 \bmod 4$, we can prove it by using the similar method.

The result of Theorem 1 shows that the linear complexity of the binary generalized cyclotomic sequence $\left(s_{i}\right)$ defined by (1) is larger than $\frac{N}{2}$, and that it can resist the Berlekamp-Massey attack.

By the definition of $\alpha, P_{1}, P_{2}, P_{3}, Q_{2}, Q_{3}, R$ and $O$, we have

$$
\begin{aligned}
& x^{p}-1=\prod_{i \in Q_{2} \cup O}\left(x-\alpha^{i}\right), \quad x^{q}-1=\prod_{i \in P_{2} \cup O}\left(x-\alpha^{i}\right) \\
& x^{r}-1=\prod_{i \in P_{1} \cup O}\left(x-\alpha^{i}\right), \quad x^{p q}-1=\prod_{i \in R \cup P_{2} \cup Q_{2} \cup O}\left(x-\alpha^{i}\right) \\
& x^{p r}-1=\prod_{i \in Q \cup O}\left(x-\alpha^{i}\right), \quad x^{q r}-1=\prod_{i \in P \cup O}\left(x-\alpha^{i}\right) . \\
& \text { Suppose } \quad d(x)=\prod_{i \in D_{0} \cup D_{1}}\left(x-\alpha^{i}\right), d_{j}(x)
\end{aligned}
$$$$
\prod_{i \in D_{j}}\left(x-\alpha^{i}\right) \text { where } j=0,1 \text {. Then } d(x)=d_{0}(x) d_{1}(x)
$$
and

$$
\begin{gathered}
x^{N}-1=\prod_{i=0}^{N-1}\left(x-\alpha^{i}\right)= \\
\frac{(x-1)\left(x^{p q}-1\right)\left(x^{p r}-1\right)\left(x^{q r}-1\right)}{\left(x^{p}-1\right)\left(x^{q}-1\right)\left(x^{r}-1\right)} d(x) .
\end{gathered}
$$

We have the following theorem.

Theorem 2 Let notations be the same as before, then the minimal polynomial $m(x)$ of the binary generalized cyclotomic sequence $\left(s_{i}\right)$ satisfies

(i) If $r \equiv 1 \bmod 4$,

$$
m(x)=\left\{\begin{array}{ll}
\frac{\left(x^{N}-1\right)\left(x^{p}-1\right)^{2}\left(x^{q}-1\right)\left(x^{r}-1\right)}{d_{0}(x)(x-1)^{3}\left(x^{p r}-1\right)\left(x^{p q}-1\right)}, & 2 \in D_{0} \\
\frac{\left(x^{N}-1\right)\left(x^{p}-1\right)^{2}\left(x^{q}-1\right)\left(x^{r}-1\right)}{(x-1)^{3}\left(x^{p r}-1\right)\left(x^{p q}-1\right)}, & 2 \in D_{1}
\end{array} .\right.
$$

(ii) If $r \equiv 3 \bmod 4$,

$$
m(x)= \begin{cases}\frac{\left(x^{N}-1\right)\left(x^{p}-1\right)\left(x^{r}-1\right)}{d_{0}(x)(x-1)^{2}\left(x^{p r}-1\right)}, & 2 \in D_{0} \\ \frac{\left(x^{N}-1\right)\left(x^{p}-1\right)\left(x^{r}-1\right)}{(x-1)^{2}\left(x^{p r}-1\right)}, & 2 \in D_{1} .\end{cases}
$$

Proof (i) If $r \equiv 1 \bmod 4$, and $2 \in D_{0}$, we have

$\operatorname{gcd}\left(x^{N}-1, S(x)\right)=\frac{d_{0}(x)(x-1)^{3}\left(x^{p r}-1\right)\left(x^{p q}-1\right)}{\left(x^{p}-1\right)^{2}\left(x^{q}-1\right)\left(x^{r}-1\right)}$.

If $r \equiv 1 \bmod 4$, and $2 \in D_{1}$, we have

$\operatorname{gcd}\left(x^{N}-1, S(x)\right)=\frac{(x-1)^{3}\left(x^{p r}-1\right)\left(x^{p q}-1\right)}{\left(x^{p}-1\right)^{2}\left(x^{q}-1\right)\left(x^{r}-1\right)}$.

(ii) If $r \equiv 3 \bmod 4$, we can prove it by using the similar method.

\section{Autocorrelation values}

By the definition of $x_{1}$ and $x_{2}$, we have $D_{i} \bmod r=D_{i}^{(r)}$ $(i=0,1)$. Hence, the sequence $\left(s_{i}\right)$ defined in (1) can be expressed as

$$
s_{i}= \begin{cases}0, & i \bmod N \in Q_{3} \cup R \cup O \\ 1, & i \bmod N \in P \cup Q_{2} \\ \frac{1-\left(\frac{i}{r}\right)}{2}, & i \bmod N \in \mathbb{Z}_{N}^{*}\end{cases}
$$

where $\left(\frac{\cdot}{r}\right)$ denotes the Legendre symbol. From (4) we get

$$
(-1)^{s_{i}}=\left\{\begin{array}{l}
1, \quad i \bmod N \in Q_{3} \cup R \cup O \\
-1, \quad i \bmod N \in P \cup Q_{2} \\
\left(\frac{i}{r}\right), \quad i \bmod N \in \mathbb{Z}_{N}^{*}
\end{array} .\right.
$$

Lemma 5 [31] Let notations be the same as before, and $\left(\frac{\cdot}{r}\right)$ denotes the Legendre symbol, then we have

$$
\begin{gathered}
\sum_{x=1}^{r-1}\left(\frac{x}{r}\right)=0 \\
\sum_{x=0}^{r-1}\left(\frac{x}{r}\right)\left(\frac{x+a}{r}\right)=-1
\end{gathered}
$$

where $\operatorname{gcd}(a, r)=1$.

Theorem 3 For the generalized cyclotomic sequence $\left(s_{i}\right)$ defined by (1), we have

(i) If $\tau \in P_{1}$,

$N \cdot A C\left(s_{i}, \tau\right)=q r-p q+(p-1)(r-3)+q-4+$

$$
\left(\frac{\tau}{r}\right)(p-1)(q-1)\left(1+(-1)^{\frac{r-1}{2}}\right)
$$

(ii) If $\tau \in P_{2}$,

$$
N \cdot A C\left(s_{i}, \tau\right)=q r+(p-1)(q-4)+
$$




$$
(p-1)(q-2)(r-1)-4 ;
$$

(iii) If $\tau \in P_{3}$,

$$
\begin{gathered}
N \cdot A C\left(s_{i}, \tau\right)=q r-p q+4(p-2)+q- \\
2\left(\frac{\tau}{r}\right)(p-1)\left(1+(-1)^{\frac{r-1}{2}}\right)+ \\
\left(\frac{\tau}{r}\right)(p-1)(q-2)\left(1+(-1)^{\frac{r-1}{2}}\right) ;
\end{gathered}
$$

(iv) If $\tau \in Q_{2}$,

$$
\begin{gathered}
N \cdot A C\left(s_{i}, \tau\right)=(p-2)(q-1)(r-1)+ \\
(p-4)(q+r-1) ;
\end{gathered}
$$

(v) If $\tau \in Q_{3}$,

$$
\begin{gathered}
N \cdot A C\left(s_{i}, \tau\right)=(p-4)(r-4)-p q+p+ \\
\left(\frac{\tau}{r}\right)(p-2)(q-1)\left(1+(-1)^{\frac{r-1}{2}}\right) ;
\end{gathered}
$$

(vi) If $\tau \in R$,

$$
\begin{gathered}
N \cdot A C\left(s_{i}, \tau\right)=(p-2)(q-2) r-2(p-2)- \\
2(q-2)-2(r-1)+4
\end{gathered}
$$

(vii) If $\tau \in \mathbb{Z}_{N}^{*}$,

$$
N \cdot A C\left(s_{i}, \tau\right)=4(p-2)-2(q-2)-2(r-2)-p q+
$$

$$
\begin{gathered}
2(q-1)-2\left(\frac{\tau}{r}\right)(p-2)\left(1+(-1)^{\frac{r-1}{2}}\right)+ \\
\left(\frac{\tau}{r}\right)(p-2)(q-2)\left(1+(-1)^{\frac{r-1}{2}}\right)+ \\
2\left(\frac{\tau}{r}\right)\left(1+(-1)^{\frac{r-1}{2}}\right) .
\end{gathered}
$$

Proof By (5) and Lemma 5,

(i) If $\tau \in P_{1}$, we have

$$
(-1)^{s_{i}+s_{i+\tau}}=\left\{\begin{array}{l}
1, \quad i \in P \backslash\{N-\tau\} \\
-1, \quad i \in\{0, N-\tau\} \\
-1, \quad i \in Q_{2} \\
-1, \quad i \in Q_{3} ; i+\tau \in Q_{2} \\
1, \quad i \in Q_{3} ; i+\tau \in Q_{3} \\
\left(\frac{\tau}{r}\right), \quad i \in R \\
\left(\frac{-\tau}{r}\right), \quad i \in \mathbb{Z}_{N}^{*} ; i+\tau \in R \\
\left(\frac{i}{r}\right)\left(\frac{i+\tau}{r}\right), \quad i \in \mathbb{Z}_{N}^{*} ; i+\tau \in \mathbb{Z}_{N}^{*}
\end{array}\right.
$$

then

$$
\begin{gathered}
N \cdot A C\left(s_{i}, \tau\right)=q r-2-2-(p-1)-(p-1)+ \\
(p-1)(r-2)+\sum_{i \in R}\left(\frac{\tau}{r}\right)+
\end{gathered}
$$

$$
\begin{gathered}
\sum_{\substack{i \in \mathbb{Z}_{N}^{*} \\
i+\tau \in R}}\left(\frac{-\tau}{r}\right)+\sum_{\substack{i \in \mathbb{Z}_{N}^{*} \\
i+\tau \in \mathbb{Z}_{N}^{*}}}\left(\frac{i}{r}\right)\left(\frac{i+\tau}{r}\right)= \\
q r-4+(p-1)(r-4)+ \\
\left(\frac{\tau}{r}\right)(p-1)(q-1)+ \\
\left(\frac{-\tau}{r}\right)(p-1)(q-1)+ \\
\left(\sum_{i \in \mathbb{Z}_{N}}-\sum_{i \in P_{1}}-\sum_{i \in P_{3}}-\sum_{i \in Q_{3}}\right)\left(\frac{i}{r}\right)\left(\frac{i+\tau}{r}\right)= \\
q r-4+(p-1)(r-4)+ \\
\left(\frac{\tau}{r}\right)(p-1)(q-1)\left(1+(-1)^{\frac{r-1}{2}}\right)- \\
p q+1+(q-1)+(p-1)= \\
q r-p q+(p-1)(r-3)+q-4+ \\
\left(\frac{\tau}{r}\right)(p-1)(q-1)\left(1+(-1)^{\frac{r-1}{2}}\right)
\end{gathered}
$$

(ii) If $\tau \in P_{2}$, we have

$$
(-1)^{s_{i}+s_{i+\tau}}=
$$

$$
\left\{\begin{array}{l}
1, \quad i \in P \backslash\{N-\tau\} \\
-1, \quad i \in\{0, N-\tau\} \\
-1, \quad i \in Q_{2} \\
\left(\frac{i}{r}\right), \quad i \in Q_{3} \\
-1, \quad i \in R ; i+\tau \in Q_{2} \\
1, \quad i \in R ; i+\tau \in R \\
\left(\frac{i}{r}\right), \quad i \in \mathbb{Z}_{N}^{*} ; i+\tau \in Q_{3} \\
\left(\frac{i}{r}\right)\left(\frac{i+\tau}{r}\right)=1, \quad i \in \mathbb{Z}_{N}^{*} ; i+\tau \in \mathbb{Z}_{N}^{*}
\end{array}\right.
$$

then

$$
\begin{gathered}
N \cdot A C\left(s_{i}, \tau\right)=q r-2-2-(p-1)+\sum_{i \in Q_{3}}\left(\frac{i}{r}\right)- \\
(p-1)+(p-1)(q-2)+ \\
\sum_{\substack{i \in \mathbb{Z}_{N}^{*} \\
i+\tau \in Q_{3}}}\left(\frac{i}{r}\right)+\sum_{\substack{i \in \mathbb{Z}_{N}^{*} \\
i+\tau \in \mathbb{Z}_{N}^{*}}} 1= \\
q r+(p-1)(q-4)+ \\
(p-1)(q-2)(r-1)-4 ;
\end{gathered}
$$

(iii) If $\tau \in P_{3}$, we have

$$
(-1)^{s_{i}+s_{i+\tau}}=
$$




$$
\left\{\begin{array}{l}
1, \quad i \in P \backslash\{N-\tau\} \\
-1, \quad i \in\{0, N-\tau\} \\
-\left(\frac{\tau}{r}\right), \quad i \in Q_{2} \\
1, \quad i \in Q_{3} ; i+\tau \in R \\
\left(\frac{i+\tau}{r}\right), \quad i \in Q_{3} ; i+\tau \in \mathbb{Z}_{N}^{*} \\
1, i \in R ; i+\tau \in Q_{3} \\
\left(\frac{\tau}{r}\right), \quad i \in R ; i+\tau \in \mathbb{Z}_{N}^{*} \\
-\left(\frac{-\tau}{r}\right), \quad i \in \mathbb{Z}_{N}^{*} ; i+\tau \in Q_{2} \\
\left(\frac{i}{r}\right), \quad i \in \mathbb{Z}_{N}^{*} ; i+\tau \in Q_{3} \\
\left(\frac{-\tau}{r}\right), \quad i \in \mathbb{Z}_{N}^{*} ; i+\tau \in R \\
\left(\frac{i}{r}\right)\left(\frac{i+\tau}{r}\right), \quad i \in \mathbb{Z}_{N}^{*} ; i+\tau \in \mathbb{Z}_{N}^{*}
\end{array}\right.
$$

then

$$
\begin{aligned}
& N \cdot A C\left(s_{i}, \tau\right)=q r-2-2-\sum_{i \in Q_{2}}\left(\frac{\tau}{r}\right)+(p-1)+ \\
& \sum_{\substack{i \in Q_{3} \\
i+\tau \in \mathbb{Z}_{N}^{*}}}\left(\frac{i+\tau}{r}\right)+(p-1)+\sum_{\substack{i \in R \\
i+\tau \in \mathbb{Z}_{N}^{*}}}\left(\frac{\tau}{r}\right)- \\
& \sum_{\substack{i \in \mathbb{Z}_{N}^{*} \\
i+\tau \in Q_{2}}}\left(\frac{-\tau}{r}\right)+\sum_{\substack{i \in \mathbb{Z}_{N}^{*} \\
i+\tau \in Q_{3}}}\left(\frac{i}{r}\right)+\sum_{\substack{i \in \mathbb{Z}_{N}^{*} \\
i+\tau \in R}}\left(\frac{-\tau}{r}\right)+ \\
& \sum_{\substack{i \in \mathbb{Z}_{N}^{*} \\
i+\tau \in \mathbb{Z}_{N}^{*}}}\left(\frac{i}{r}\right)\left(\frac{i+\tau}{r}\right)= \\
& q r-4-\left(\frac{\tau}{r}\right)(p-1)+(p-1)+ \\
& (p-1)\left(\sum_{i \in \mathbb{Z}_{r}}\left(\frac{i}{r}\right)-\left(\frac{\tau}{r}\right)\right)+(p-1)+ \\
& \left(\frac{\tau}{r}\right)(p-1)(q-2)-\left(\frac{-\tau}{r}\right)(p-1)+ \\
& (p-1)\left(\sum_{i \in \mathbb{Z}_{r}}\left(\frac{i}{r}\right)-\left(\frac{-\tau}{r}\right)\right)+ \\
& \left(\frac{-\tau}{r}\right)(p-1)(q-2)+ \\
& \left(\sum_{i \in \mathbb{Z}_{N}}-\sum_{i \in P_{1}}-\sum_{i \in P_{3}}-\sum_{i \in Q_{3}}-\sum_{i+\tau \in Q_{3}}\right)\left(\frac{i}{r}\right)\left(\frac{i+\tau}{r}\right)= \\
& q r-p q+4(p-2)+q- \\
& 2\left(\frac{\tau}{r}\right)(p-1)\left(1+(-1)^{\frac{r-1}{2}}\right)+
\end{aligned}
$$

$$
\left(\frac{\tau}{r}\right)(p-1)(q-2)\left(1+(-1)^{\frac{r-1}{2}}\right)
$$

(iv) If $\tau \in Q_{2}$, we have

$$
(-1)^{s_{i}+s_{i+\tau}}=
$$

$$
\left\{\begin{array}{l}
-1, \quad i \in P_{1} \\
-1, \quad i \in P_{2} \\
-\left(\frac{i}{r}\right), \quad i \in P_{3} \\
1, \quad i \in Q_{2} \backslash\{N-\tau\} \\
-1, \quad i \in\{0, N-\tau\} \\
-1, \quad i \in Q_{3} ; i+\tau \in P_{1} \\
1, \quad i \in Q_{3} ; i+\tau \in Q_{3} \\
-1, \quad i \in R ; i+\tau \in P_{2} \\
1, \quad i \in R ; i+\tau \in R \\
-\left(\frac{i}{r}\right), \quad i \in \mathbb{Z}_{N}^{*} ; i+\tau \in P_{3} \\
\left(\frac{i}{r}\right)\left(\frac{i+\tau}{r}\right)=1, \quad i \in \mathbb{Z}_{N}^{*} ; i+\tau \in \mathbb{Z}_{N}^{*}
\end{array}\right.
$$

then

$$
\begin{gathered}
N \cdot A C\left(s_{i}, \tau\right)=-(r-1)-(q-1)-\sum_{i \in P_{3}}\left(\frac{i}{r}\right)+p-2- \\
2-(r-1)+(p-2)(r-1)-(q-1)+ \\
(p-2)(q-1)-\sum_{\substack{i \in \mathbb{Z}_{N}^{*} \\
i+\tau \in P_{3}}}\left(\frac{i}{r}\right)+\sum_{\substack{i \in \mathbb{Z}_{N}^{*} \\
i+\tau \in \mathbb{Z}_{N}^{*}}} 1= \\
(p-2)(q-1)(r-1)+(p-4)(q+r-1) ;
\end{gathered}
$$

(v) If $\tau \in Q_{3}$, we have

$$
(-1)^{s_{i}+s_{i+\tau}}=\left\{\begin{array}{l}
1, \quad i \in P_{1} ; i+\tau \in Q_{2} \\
-1, \quad i \in P_{1} ; i+\tau \in Q_{3} \\
-\left(\frac{\tau}{r}\right), \quad i \in P_{2} \\
-1, \quad i \in P_{3} ; i+\tau \in R \\
-\left(\frac{i+\tau}{r}\right), \quad i \in P_{3} ; i+\tau \in \mathbb{Z}_{N}^{*} \\
1, \quad i \in Q_{2} ; i+\tau \in P_{1} \\
-1, \quad i \in Q_{2} ; i+\tau \in Q_{3} \\
-1, \quad i \in Q_{3} \backslash\{N-\tau\} ; i+\tau \in P_{1} \\
-1, \quad i \in Q_{3} \backslash\{N-\tau\} ; i+\tau \in Q_{2} \\
1, \quad i \in Q_{3} \backslash\{N-\tau\} ; i+\tau \in Q_{3} \\
1, \quad i \in\{0, N-\tau\} \\
-1, \quad i \in R ; i+\tau \in P_{3}
\end{array}\right.
$$




$$
(-1)^{s_{i}+s_{i+\tau}}=\left\{\begin{array}{l}
\left(\frac{\tau}{r}\right), \quad i \in R ; i+\tau \in \mathbb{Z}_{N}^{*} \\
-\left(\frac{-\tau}{r}\right), \quad i \in \mathbb{Z}_{N}^{*} ; i+\tau \in P_{2} \\
-\left(\frac{i}{r}\right), \quad i \in \mathbb{Z}_{N}^{*} ; i+\tau \in P_{3} \\
\left(\frac{-\tau}{r}\right), \quad i \in \mathbb{Z}_{N}^{*} ; i+\tau \in R \\
\left(\frac{i}{r}\right)\left(\frac{i+\tau}{r}\right), \quad i \in \mathbb{Z}_{N}^{*} ; i+\tau \in \mathbb{Z}_{N}^{*}
\end{array}\right.
$$

then

$$
\begin{aligned}
& N \cdot A C\left(s_{i}, \tau\right)=1-(r-2)-\left(\frac{\tau}{r}\right)(q-1)- \\
& (q-1)-\sum_{\substack{i \in P_{3} \\
i+\tau \in \mathbb{Z}_{N}^{*}}}\left(\frac{i+\tau}{r}\right)+1- \\
& (p-2)-(r-2)-(p-2)+ \\
& (p-2)(r-2)+2-(q-1)+ \\
& \left(\frac{\tau}{r}\right)(p-2)(q-1)-\left(\frac{-\tau}{r}\right)(q-1)- \\
& \sum_{\substack{i \in \mathbb{Z}_{N}^{*} \\
i+\tau \in P_{3}}}\left(\frac{i}{r}\right)+\left(\frac{-\tau}{r}\right)(p-2)(q-1)+ \\
& \sum_{\substack{i \in \mathbb{Z}_{N}^{*} \\
i+\tau \in \mathbb{Z}_{N}^{*}}}\left(\frac{i}{r}\right)\left(\frac{i+\tau}{r}\right)= \\
& (p-4)(r-4)-p q+p+ \\
& \left(\frac{\tau}{r}\right)(p-2)(q-1)\left(1+(-1)^{\frac{r-1}{2}}\right) ;
\end{aligned}
$$

(vi) If $\tau \in R$, we have

$$
(-1)^{s_{i}+s_{i+\tau}}=\left\{\begin{array}{l}
-\left(\frac{i}{r}\right), \quad i \in P_{1} \\
1, \quad i \in P_{2} ; i+\tau \in Q_{2} \\
-1, \quad i \in P_{2} ; i+\tau \in R \\
-1, \quad i \in P_{3} ; i+\tau \in Q_{3} \\
-\left(\frac{i}{r}\right), \quad i \in P_{3} ; i+\tau \in \mathbb{Z}_{N}^{*} \\
1, \quad i \in Q_{2} ; i+\tau \in P_{2} \\
-1, \quad i \in Q_{2} ; i+\tau \in R \\
-1, \quad i \in Q_{3} ; i+\tau \in P_{3} \\
\left(\frac{i}{r}\right), \quad i \in Q_{3} ; i+\tau \in \mathbb{Z}_{N}^{*} \\
-1, \quad i \in R \backslash\{N-\tau\} ; i+\tau \in P_{2} \\
-1, \quad i \in R \backslash\{N-\tau\} ; i+\tau \in Q_{2} \\
1, \quad i \in R \backslash\{N-\tau\} ; i+\tau \in R \\
1, \quad i \in\{0, N-\tau\}
\end{array}\right.
$$

$$
(-1)^{s_{i}+s_{i+\tau}}=\left\{\begin{array}{l}
-\left(\frac{i}{r}\right), \quad i \in \mathbb{Z}_{N}^{*} ; i+\tau \in P_{1} \\
-\left(\frac{i}{r}\right), \quad i \in \mathbb{Z}_{N}^{*} ; i+\tau \in P_{3} \\
\left(\frac{i}{r}\right), \quad i \in \mathbb{Z}_{N}^{*} ; i+\tau \in Q_{3} \\
1, \quad i \in \mathbb{Z}_{N}^{*} ; i+\tau \in \mathbb{Z}_{N}^{*}
\end{array}\right.
$$

then

$$
N \cdot A C\left(s_{i}, \tau\right)=-\sum_{i \in P_{1}}\left(\frac{i}{r}\right)+1-(q-2)-(r-1)-
$$

$$
\sum_{\substack{i \in P_{3} \\ i+\tau \in \mathbb{Z}_{N}^{*}}}\left(\frac{i}{r}\right)+1-(p-2)-(r-1)+
$$$$
\sum_{\substack{i \in Q_{3} \\ i+\tau \in \mathbb{Z}_{N}^{*}}}\left(\frac{i}{r}\right)-(q-2)-(p-2)+
$$$$
(p-2)(q-2)+2-\sum_{\substack{i \in \mathbb{Z}_{N}^{*} \\ i+\tau \in P_{1}}}\left(\frac{i}{r}\right)-
$$$$
\sum_{\substack{i \in \mathbb{Z}_{N}^{*} \\ i+\tau \in P_{3}}}\left(\frac{i}{r}\right)+\sum_{\substack{i \in \mathbb{Z}_{N}^{*} \\ i+\tau \in Q_{3}}}\left(\frac{i}{r}\right)+\sum_{\substack{i \in \mathbb{Z}_{N}^{*} \\ i+\tau \in \mathbb{Z}_{N}^{*}}} 1=
$$$$
(p-2)(q-2) r-2(p-2)-
$$$$
2(q-2)-2(r-1)+4
$$

(vii) If $\tau \in \mathbb{Z}_{N}^{*}$, we have

$$
(-1)^{s_{i}+s_{i+\tau}}=
$$

$$
\left\{\begin{array}{l}
-1, \quad i \in P_{1} ; i+\tau \in R \\
-\left(\frac{i+\tau}{r}\right), \quad i \in P_{1} ; i+\tau \in \mathbb{Z}_{N}^{*} \\
-1, \quad i \in P_{2} ; i+\tau \in Q_{3} \\
-\left(\frac{\tau}{r}\right), \quad i \in P_{2} ; i+\tau \in \mathbb{Z}_{N}^{*}
\end{array}\right.
$$

1, $i \in P_{3} ; i+\tau \in Q_{2}$

$-1, \quad i \in P_{3} ; i+\tau \in Q_{3}$

$-1, \quad i \in P_{3} ; i+\tau \in R$

$-\left(\frac{i+\tau}{r}\right), \quad i \in P_{3} ; i+\tau \in \mathbb{Z}_{N}^{*}$

$1, \quad i \in Q_{2} ; i+\tau \in P_{3}$

$-\left(\frac{\tau}{r}\right), \quad i \in Q_{2} ; i+\tau \in \mathbb{Z}_{N}^{*}$

$-1, \quad i \in Q_{3} ; i+\tau \in P_{2}$

$-1, \quad i \in Q_{3} ; i+\tau \in P_{3}$

$1, \quad i \in Q_{3} ; i+\tau \in R$

$\left(\frac{i+\tau}{r}\right), \quad i \in Q_{3} ; i+\tau \in \mathbb{Z}_{N}^{*}$

$-1, \quad i \in R ; i+\tau \in P_{1}$

$-1, \quad i \in R ; i+\tau \in P_{3}$

(1, $i \in R ; i+\tau \in Q_{3}$ 


$$
\left\{\begin{array}{l}
\left(\frac{\tau}{r}\right), \quad i \in R ; i+\tau \in \mathbb{Z}_{N}^{*} \\
-\left(\frac{i}{r}\right), \quad i \in \mathbb{Z}_{N}^{*} \backslash\{N-\tau\} ; i+\tau \in P_{1} \\
-\left(\frac{-\tau}{r}\right), \quad i \in \mathbb{Z}_{N}^{*} \backslash\{N-\tau\} ; i+\tau \in P_{2} \\
-\left(\frac{i}{r}\right), \quad i \in \mathbb{Z}_{N}^{*} \backslash\{N-\tau\} ; i+\tau \in P_{3} \\
-\left(\frac{-\tau}{r}\right), \quad i \in \mathbb{Z}_{N}^{*} \backslash\{N-\tau\} ; i+\tau \in Q_{2} \\
\left(\frac{i}{r}\right), \quad i \in \mathbb{Z}_{N}^{*} \backslash\{N-\tau\} ; i+\tau \in Q_{3} \\
\left(\frac{-\tau}{r}\right), \quad i \in \mathbb{Z}_{N}^{*} \backslash\{N-\tau\} ; i+\tau \in R \\
\left(\frac{i}{r}\right)\left(\frac{i+\tau}{r}\right), \quad i \in \mathbb{Z}_{N}^{*} \backslash\{N-\tau\} ; i+\tau \in \mathbb{Z}_{N}^{*} \\
\left(\frac{\tau}{r}\right), \quad i=0 \\
\left(\frac{-\tau}{r}\right), \quad i=N-\tau
\end{array}\right.
$$

then

$$
\begin{aligned}
& N \cdot A C\left(s_{i}, \tau\right)=-1-\sum_{\substack{i \in P_{1} \\
i+\tau \in \mathbb{Z}_{N}^{*}}}\left(\frac{i+\tau}{r}\right)-1-\left(\frac{\tau}{r}\right)(q-2)+ \\
& 1-(r-2)-(q-2)-\sum_{\substack{i \in P_{3} \\
i+\tau \in \mathbb{Z}_{N}^{*}}}\left(\frac{i+\tau}{r}\right)+1- \\
& \left(\frac{\tau}{r}\right)(p-2)-1-(r-2)+(p-2)+ \\
& \sum_{\substack{i \in Q_{3} \\
i+\tau \in \mathbb{Z}_{N}^{*}}}\left(\frac{i+\tau}{r}\right)-1-(q-2)+(p-2)+ \\
& \left(\frac{\tau}{r}\right)(p-2)(q-2)-\sum_{\substack{i \in \mathbb{Z}_{N}^{*} \backslash\{N-\tau\} \\
i+\tau \in P_{1}}}\left(\frac{i}{r}\right)- \\
& \left(\frac{-\tau}{r}\right)(q-2)-\sum_{\substack{i \in \mathbb{Z}_{N}^{*} \backslash\{N-\tau\} \\
i+\tau \in P_{3}}}\left(\frac{i}{r}\right)- \\
& \left(\frac{-\tau}{r}\right)(p-2)+\sum_{\substack{i \in \mathbb{Z}_{N}^{*} \backslash\{N-\tau\} \\
i+\tau \in Q_{3}}}\left(\frac{i}{r}\right)+ \\
& \left(\frac{-\tau}{r}\right)(p-2)(q-2)+
\end{aligned}
$$

$$
\begin{gathered}
\sum_{\substack{i \in \mathbb{Z}_{N}^{*} \backslash\{N-\tau\} \\
i+\tau \in \mathbb{Z}_{N}^{*}}}\left(\frac{i}{r}\right)\left(\frac{i+\tau}{r}\right)+\left(\frac{\tau}{r}\right)+\left(\frac{-\tau}{r}\right)= \\
4(p-2)-2(q-2)-2(r-2)-p q+ \\
2(q-1)-2\left(\frac{\tau}{r}\right)(p-2)\left(1+(-1)^{\frac{r-1}{2}}\right)+ \\
\left(\frac{\tau}{r}\right)(p-2)(q-2)\left(1+(-1)^{\frac{r-1}{2}}\right)+ \\
2\left(\frac{\tau}{r}\right)\left(1+(-1)^{\frac{r-1}{2}}\right)
\end{gathered}
$$

and the result follows.

Theorem 4 Let notations be the same as before.

(i) If $p \bmod r \in D_{0}^{(r)}, \tau \in P_{30}$, or $p \bmod r \in$ $D_{1}^{(r)}, \tau \in P_{31}$, we have $\left(\frac{\tau}{r}\right)=1$.

If $p \bmod r \in D_{0}^{(r)}, \tau \in P_{31}$, or $p \bmod r \in D_{1}^{(r)}, \tau \in$ $P_{30}$, we have $\left(\frac{\tau}{r}\right)=-1$.

(ii) If $q \bmod r \in D_{0}^{(r)}, \tau \in Q_{30}$, or $q \bmod r \in$ $D_{1}^{(r)}, \tau \in Q_{31}$, we have $\left(\frac{\tau}{r}\right)=1$.

If $q \bmod r \in D_{0}^{(r)}, \tau \in Q_{31}$, or $q \bmod r \in D_{1}^{(r)}, \tau \in$ $Q_{30}$, we have $\left(\frac{\tau}{r}\right)=-1$.

(iii) If $p q \bmod r \in D_{0}^{(r)}, \tau \in P_{10}$, or $p q \bmod r \in$ $D_{1}^{(r)}, \tau \in P_{11}$, we have $\left(\frac{\tau}{r}\right)=1$.

If $p q \bmod r \in D_{0}^{(r)}, \tau \in P_{11}$, or $p q \bmod r \in$ $D_{1}^{(r)}, \tau \in P_{10}$, we have $\left(\frac{\tau}{r}\right)=-1$.

(iv) If $\tau \in D_{0}$, we have $\left(\frac{\tau}{r}\right)=1$.

If $\tau \in D_{1}$, we have $\left(\frac{\tau}{r}\right)=-1$.

From Theorem 4, the exact value of the autocorrelation function of the new binary generalized cyclotomic sequences $\left(s_{i}\right)$ can be determined. By Theorem 3 and Theorem 4, if $\tau \in P_{1}$, the autocorrelation values of $\left(s_{i}\right)$ satisfy $\frac{p r+q r-3 p q}{p q r} \leqslant A C\left(s_{i}, \tau\right) \leqslant \frac{p r+q r+p q}{p q r}$, which is close to 0 if $r$ is a big prime. If $\tau \in P_{2}$, the autocorrelation values of $\left(s_{i}\right)$ satisfy $A C\left(s_{i}, \tau\right) \leqslant 1$. In other cases, we can use the same method as above to determine the autocorrelation values which is close to 0 or 1 .

Table 1 shows the difference between the proposed sequences and the sequences in [27]-[29]. $L C_{\max }$ is the maximum value that the linear complexity of the sequence can achieve, $\times$ indicates the autocorrelation of the sequences were not provided in the corresponding studies. 
Table 1 Difference between our sequences and the sequences in [27] - [29]

\begin{tabular}{cccc}
\hline Sequence & Balance & $L C_{\max }$ & $A C$ \\
\hline$[27]$ & $|(p-1)(q+r-1)-q r+2|$ & $p q r-(p-1) r-1$ & $\times$ \\
{$[28]$} & $|(p-1)(q+r-3)-(q-1)(r-1)-1|$ & $p q r-(p-1)(q-1)-1$ & $\times$ \\
{$[29]$} & $\left|\left(p_{1}-1\right)\left(p_{2}+p_{3}-1\right)-p_{2} p_{3}+2\right|$ & $p_{1} p_{2} p_{3}-p_{1}$ & $\times$ \\
Ours & $|(p-1)(q+r-3)-q r+2|$ & $p q r-(p-1)(r-1)-1$ & $\sqrt{ }$ \\
\hline
\end{tabular}

\section{Simulations}

Based on the tools $\mathrm{C}++$ and Matlab, Table 2 lists the exact linear complexities of $\left(s_{i}\right)$ by the Berlekamp-Massey algorithm and linear complexities of $\left(s_{i}\right)$ given by Theorem 1 . The conclusion about the linear complexity of $\left(s_{i}\right)$ in Theorem 1 is correct. Table 3 and Table 4 give the exact autocorrelation values of $\left(s_{i}\right)$ by the definition of the autocorrelation function $A C\left(s_{i}, \tau\right)$ and autocorrelation values of $\left(s_{i}\right)$ given by Theorem 3 when $r \equiv 3 \bmod 4$ and $r \equiv$ $1 \bmod 4$, respectively. The autocorrelation values of $\left(s_{i}\right)$ in Theorem 3 are the same as the tested values. Fig. 1 and Fig. 2 show that the autocorrelation values of $\left(s_{i}\right)$ when $p=19$, are $q=11, r=3$ and $p=19, q=11, r=5$, respectively.

Table 2 Linear complexity of the new sequences $\left(s_{i}\right)$

\begin{tabular}{cccccc}
\hline$(p, q, r)$ & $g$ & $2 \in D_{1}$ & $t$ & $L C\left(s_{i}\right)$ & Our results \\
\hline$(19,11,3)$ & 2 & Yes & 627 & 590 & 590 \\
$(19,11,5)$ & 2 & Yes & 1045 & 792 & 792 \\
$(47,31,23)$ & 11 & No & 33511 & 17318 & 17318 \\
$(43,31,17)$ & 3 & No & 22661 & 10648 & 10648 \\
\hline
\end{tabular}

Table 3 Autocorrelation values of $\left(s_{i}\right)$

\begin{tabular}{ccc}
\hline$(479,383,227)$ & $A C\left(s_{i}, \tau\right)$ & Our results \\
\hline$\tau \in P_{1}$ & 0.000263 & 0.000263 \\
$\tau \in P_{2}$ & 0.994766 & 0.994766 \\
$\tau \in P_{3}$ & -0.002263 & -0.002263 \\
$\tau \in Q_{2}$ & 0.995795 & 0.995795 \\
$\tau \in Q_{3}$ & -0.001850 & -0.001850 \\
$\tau \in R$ & 0.990573 & 0.990573 \\
$\tau \in \mathbb{Z}_{N}^{*}$ & -0.004370 & -0.004370 \\
\hline
\end{tabular}

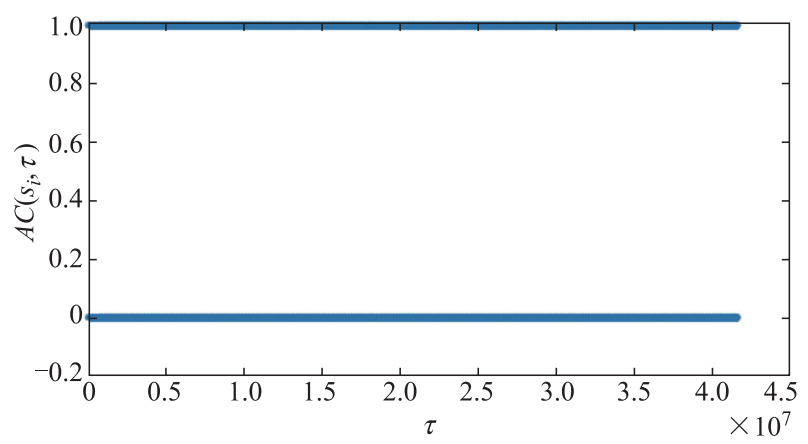

Fig. 1 Autocorrelation values of $p=479, q=383, r=227$

Table 2 shows that the linear complexity of $\left(s_{i}\right)$ is high when $2 \in D_{1}$. By Table 3 and Table 4 , the autocorrelation value of the new sequences is close to 1 when $\tau \in P_{2} \cup Q_{2} \cup R$, and close to 0 in other cases. The cardinality of $P_{2} \cup Q_{2} \cup R$ approximates $p q$; therefore, the autocorrelation values are close to 0 with a probability of approximately $\left(1-\frac{1}{r}\right)$. Thus, when $r$ is a big prime, the new sequence has a good autocorrelation.

Table 4 Autocorrelation values of $\left(s_{i}\right)$

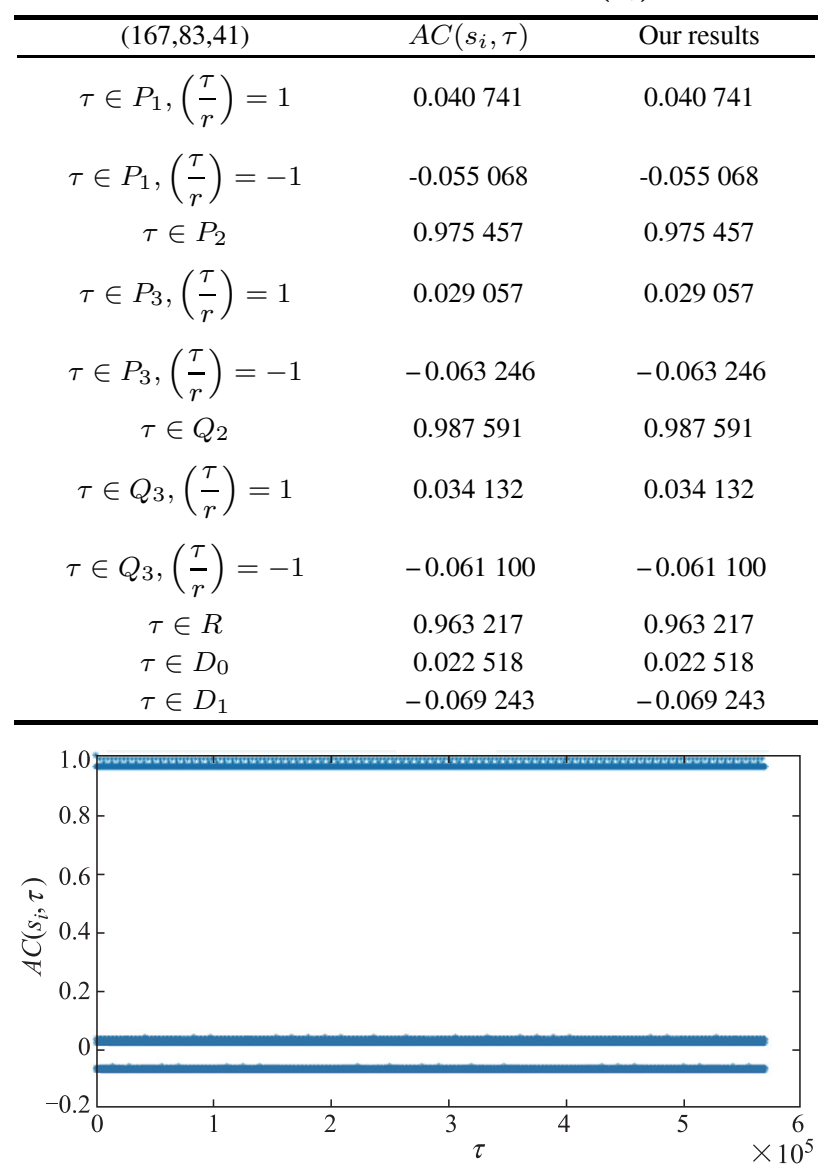

Fig. 2 Autocorrelation values of $p=167, q=83, r=41$

\section{Conclusions}

In this paper, we determine the linear complexity and the autocorrelation values of the new binary generalized cyclotomic sequences $\left(s_{i}\right)$. The results show that the linear complexity of the sequence is high when $2 \in D_{1}$. Furthermore, the autocorrelation of the sequence is close to 1 when $\tau \in P_{2} \cup Q_{2} \cup R$, and the autocorrelation is close to 0 at other points. Therefore, the new sequence has a good autocorrelation when $r$ is a big prime. 


\section{References}

[1] TURYN R J. The linear generation of the legendre sequence. Journal of the Society for Industrial and Applied Mathematics, 1964, 12(1): $115-116$.

[2] DING C, HESSESETH T, SHAN W. On the linear complexity of Legendre sequences. IEEE Trans. on Information Theory, 1998, 44(3): 1276-1278.

[3] STANTON R G, SPROTT D A. A family of difference sets. Canadian Journal of Mathematics, 1958, 6(1): 73-77.

[4] DING C. Binary cyclotomic generators. Lecture Notes in Computer Science, 1994, 1008: 29-60.

[5] DING C . Linear complexity of generalized cyclotomic binary sequences of order 2. Finite Fields and Their Applications, 1997, 3(2): 159 - 174.

[6] DING C. Autocorrelation values of generalized cyclotomic sequences of order two. IEEE Trans. on Information Theory, 1998, 44(4): $1699-1702$.

[7] BAI E, FU X, XIAO G. On the linear complexity of generalized cyclotomic sequences of order four over $\mathbb{Z}_{p q}$. IEICE Trans. on Fundamentals, 2005, 88(1): 392-395.

[8] BRANDSTÄTTER N, WINTERHOF A. Some notes of the two-prime generator of order 2. IEEE Trans. on Information Theory, 2005, 51(10): $3654-3657$.

[9] DING C, HELLESETH T. New generalized cyclotomy and its application. Finite Fields and Their Applications, 1998, 4(2): $140-166$.

[10] BAI E, LIU X. Generalized cyclotomic sequences of order four over $\mathbb{Z}_{p q}$ and their autocorrelation values. Chinese Journal of Engineering Mathematics, 2008, 25(5): 894-900. (In Chinese)

[11] BAI E, LIU X, XIAO G. Lilnear complexity of new generelized cyclotomic sequences of order two of length $p q$. IEEE Trans. on Information Theory, 2005, 51(5): 1849-1853.

[12] YAN T, HONG L, XIAO G. The linear complexity of new generalized cyclotomic binary sequences of order four. Information Sciences, 2008, 178(3): 807-815.

[13] JIN S, KIM Y, SONG H. Autocorrelation of new generalized cyclotomic sequences of period $p^{n}$. IEICE Trans. on Fundamentals, 2010, 93(11): 2345-2348.

[14] KE P, ZHANG J, ZHANG S. On the linear complexity and the autocorrelation of generalized cyclotomic binary sequences of length $2 p^{m}$. Designs, Codes and Cryptography, 2013, 67(3): $325-339$.

[15] LI S, CHEN Z, SUN R, et al. On the randomness of generalized cyclotomic sequences of order two and length $p q$. IEICE Trans. on Fundamentals, 2007, 90(9): 2037-2041.

[16] LI S, CHEN Z, FU X, et al. Autocorrelation values of new generalized cyclotomic sequences of order two and length $p q$. Journal of Computer Science and Technology, 2007, 22(6): $830-834$

[17] Meidl W. Remarks on a cyclotomic sequence. Designs, Codes and Cryptography, 2009, 51(1): $33-43$.

[18] YAN T, SUN R, XIAO G. Autocorrelation and linear complexity of the new generalized cyclotomic sequences. IEICE Trans. on Fundamentals, 2008, 90(4): 857-864.

[19] YAN T, DU X, XIAO G, et al. Linear complexity of binary Whiteman generalized cyclotomic sequences of order $2 k$. Information Sciences, 2009, 179(7): 1019-1023.

[20] YAN T, HUANG B, XIAO G. Cryptographic properties of some binary generalized cyclotomic sequences with the length $p^{2}$. Information Sciences, 2008, 178(4): 1078-1086.

[21] YAN T, LI S, XIAO G. On the linear complexity of generalized cyclotomic sequences with the period $p^{m}$. Appliced Mathematics Letters, 2008, 21(2): 187-193.

[22] YAN T, CHEN Z, XIAO G. Linear complexity of Ding generalized cyclotomic sequences. Journal of Shanghai University, 2007, 11(1): $22-26$.

[23] ZHANG J, ZHAO C, MA X. Linear complexity of generalized cyclotomoc binary sequences of length $2 p^{m}$. Applicable Algebra in Engineering, Communication and Computing, 2010, 21(2): $93-108$.

[24] CHEN Z, DU X, WU C. Pseodu-randomness of certain sequences of $k$ symbols with length $p q$. Journal of Computer Science and Technology, 2011, 26(2): 276-282.

[25] CHEN Z, DU X. Linear complexity and auto- correlation values of a polyphase generalized cyclotomic sequence of length $p q$. Frontiers of Computer Science, 2010, 4(4): 529-535.

[26] DING C, HELLESETH T. On cyclotomic generator of order $r$. Information Processing Letters, 1998, 66(1): 21-25.

[27] CHANG Z, ZHOU Y, KE P. Linear complexity of new generalized cyclotomic sequences of order two and length pqr. Acta Electronica Science, 2015, 43(1): 166 - 170. (In Chinese)

[28] LIU L, YANG X, DU X, et al. On the linear complexity of new generalized cyclotomic binary sequences of order two and period $p q r$. Tsinghua Science and Technology, 2016, 21(3): $295-301$.

[29] LYU C, XIAO G Z, YAN T J. On linear complexity of a new generalized cyclotmic sequence of order two over $\mathbb{Z}_{p_{1} p_{2} p_{3}}$. Journal of Computational Information Systems, 2015, 11(17): $6355-6362$.

[30] CUSICK T W, DING C, RENVALL A. Stream cipher and number theory. Amsterdam: North-Holland, 1988.

[31] LIDL R, NIEDERREITER H. Finite fields. 2nd ed. Cambridge: Cambridge University Press, 1997.

\section{Biographies}

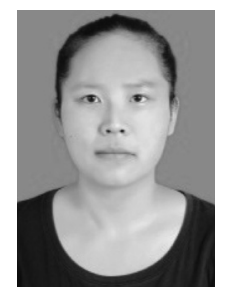

JIA Wenjuan was born in 1994. She received her B.S. degree from Northwest Normal University. She is a Ph.D candidate in cryptography from Xidian University. Her research interests include Latticebased cryptography, stream cipher and pseudorandom sequence.

E-mail: 18368910175@163.com

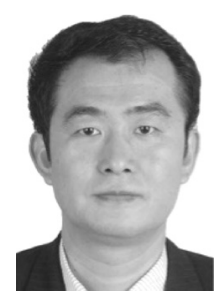

GAO Juntao was born in 1979. He received his D.E. degree in cryptography from Xidian University. $\mathrm{He}$ is now an associate professor. His research interests include block chain, stream cipher and pseudorandom sequence.

E-mail: jtgao@mail.xidian.edu.cn

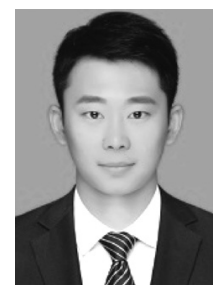

ZHANG Peng was born in 1992. He received his B.S. degree from Henan University and his M.S. degree in cryptography from Xidian University. His research interests include compressive sensing and cryptography.

E-mail: 297966738@qq.com 